\title{
Errata for Vol. 7
}

Page Line, heading, etc. Error Correction

No. 1

19 REFERENCES $\downarrow$ 14-15

$25 \downarrow 3$, Table 3

$26 \downarrow 5$

No. 2

$141 \downarrow 8$

$144 \downarrow 15$

$149 \downarrow 1$

$162 \downarrow 5$

166 footnote of Table $6, \uparrow 1$

$170 \uparrow 7$

170 footnote 20

$173 \uparrow 5$

$173 \uparrow 5$

$181 \downarrow 13$

$193 \downarrow 8$

$193 \downarrow 9$

$194 \uparrow 10$

$194 \uparrow 9$

$199 \downarrow 4$

203 REFERENCES $\downarrow 13$

$227 \uparrow 5$

249 Fig. 25c.

No. 3

389 running title

No. 4

$408 \downarrow 13$

$408 \downarrow 14$

410 legend to Fig. $5, \downarrow 1$

413 Table 2, column of $\mathrm{M}_{2}$

$414 \downarrow 1$

415 Table 5, column of $\mathrm{M}^{2}$

416 Table 8 , column of $\mathrm{P} 4$

CONTENTS OF VOL. 7, No. 2
Kandoll

KANDOLL

field adult

Takasakiyama

juvenile

infants

was in

her

age estimated

peripheral

adout

peripheralization

solitarization

in the females

higher status

mature

Fig. 10a.

the

\begin{tabular}{ll}
$\begin{array}{ll}\text { five cusps } \\
\text { four cusps, }\end{array}$ & five main cusps \\
molar & four main cusps, \\
0 & molars \\
molar & 10 \\
4 & molars \\
+0.96 & 14 \\
Aloutta & -0.96 \\
\hline
\end{tabular}

of

delete lines 14-15 and insert after line 11

Kendall

KENDALL

field some adult

Takagoyama

peripheral

juveniles

was not in

his

age was estimated

solitary

delete "solitary males and"

about

solitarization

peripheralization

in the separation of the females

lower status from the troop.

delete "three"

nature

Figs. $10 \mathrm{a}, 10 \mathrm{~b}, \& 10 \mathrm{c}$.

top and bottom should be reversed.

Alouatta 